Received: 2013.07.25 Accepted: 2013.08.08 Published: 2013.10.28

Authors' Contribution: Study Design A Data Collection B Statistical Analysis C Data Interpretation D Manuscript Preparation E Literature Search $F$ Funds Collection G
ABCDEF Chad J. Cooper

CDEF Sarmad Said

DEF Angelica Nunez

DEF Raphael Quansah

DEF Sayeed Khalillullah

DEF German T. Hernandez
Department of Internal Medicine,Texas Tech University Health Sciences Center, El Paso, TX, U.S.A.

Patient: Male, 32

Final Diagnosis:

Symptoms:

Medication:

Clinical Procedure:

Specialty:

Objective:

Background:

Case Report:

Conclusions:

Key words:

Full-text PDF:
Dural arterio-venous fistula

Eye redness $\bullet$ post-trauma headache $\bullet$ tinnitus

\section{Fistula embolization}

Neurology

\section{Mistake in diagnosis}

A dural arteriovenous fistula (DAVF), is an abnormal direct connection (fistula) between a meningeal artery and a meningeal vein or dural venous sinus. The pathogenesis of DAVF still remains unclear. Sinus thrombosis, head trauma, chronic central nervous system, hypercoagulable state, surgery, and hormonal influence are the predisposing factors that initiate this disease. The symptoms experienced by the patient will depend on the location of the fistula.

Thirty-two year old Hispanic male who presented one day after a rear ended motor vehicle collision (MVC) with a severe throbbing headache in the left parietal region, left eye redness but no retro-orbital pain and tinnitus in the left ear. He was initially misdiagnosed to have a carotid-cavernous fistula but upon cerebral angiogram was actually diagnosed with a dural arterio-venous fistula in the posterior fossa venous system followed by successful embolization of the fistula.

A cerebral angiography is the gold standard for detection and characterization of a DAVF and will distinguish it from a CCF. Endovascular surgery involves a catheter-based technique for embolization of the lumen of arteries feeding the DAVF, or directly into the vein draining the DAVF. It is very important to recognize the typical findings of patients presenting with a DAVF then quickly proceeding with a cerebral angiogram to determine the exact location of the fistula and the appropriate treatment plan. By diagnosing and treating a DAVF as early as possible, the associated fatal complications can be averted.

dural arteriovenous fistula • cerebral angiography • endovascular embolization • trauma

http://www.amjcaserep.com/download/index/idArt/889610 


\section{Background}

A dural arteriovenous fistula (DAVF), is an abnormal direct connection (fistula) between a meningeal artery and a meningeal vein or dural venous sinus. Ten to $15 \%$ of intracranial arteriovenous fistulas are most commonly related to dural venous sinuses [1]. Classic DAVFs are situated within the dura adjacent to the venous sinuses, such as the transverse, sigmoid, and cavernous sinuses [2]. Cranial DAVF is supplied by branches of the carotid artery (external and internal carotid arteries) and possibly also by branches of the vertebral artery before they penetrate the dura. The fistula usually resides in the convexity of the duramater overlying the brain hemisphere or in the tentorial dura between the forebrain and hindbrain [3]. Rarely, it lies in a deeper region of the brain especially the cavernous sinus.

The Borden Classification of dural arteriovenous fistulas establishes that there are three types of DAVF based upon their venous drainage. Type 1: the dural arterial supply (meningeal artery) drains anterograde into meningeal vein or dural venous sinus. Type la: a simple dural arteriovenous fistulas have a single meningeal arterial supply. Type Ib: a more complex arteriovenous fistulas are supplied by multiple meningeal arteries. Type 2: the dural arterial supply drains into venous sinus. The high pressure within a type 2 fistula causes the blood to flow in a retrograde fashion in the subarachnoid veins that drain into the sinus. Type 3: the dural arterial supply drains retrograde into subarachnoid veins. The arterial supply in each of these three types is derived from meningeal arteries [4].

Frequently the blood flow in a DAVF is very high, and this may cause the blood to flow in a retrograde direction, which can comprise of abnormal cortical venous drainage. Although DAVF are very rare they are more common over the age of 40 years (Cranial DAVF are more in women, while spinal DAVF are more common in men). The most common cause of a dural fistula is thrombotic occlusion with subsequent recanalization [5]. The common predisposing factor for DAVFs appears to be venous sinus thrombosis. The venous hypertension developing after venous thrombosis causes microvascular connections within the dura [6]. These connections then result in direct shunting between the arteries and veins. The dural venous sinus takes venous blood from the brain towards the base of the brain to form the internal jugular vein. If the venous sinus is blocked off then abnormal collateral pathways are created in the form of a fistula. The pathogenesis of DAVF still remains unclear. Sinus thrombosis, head trauma, chronic central nervous system, hypercoagulable state, surgery, and hormonal influence are the predisposing factors that initiate this disease [7].

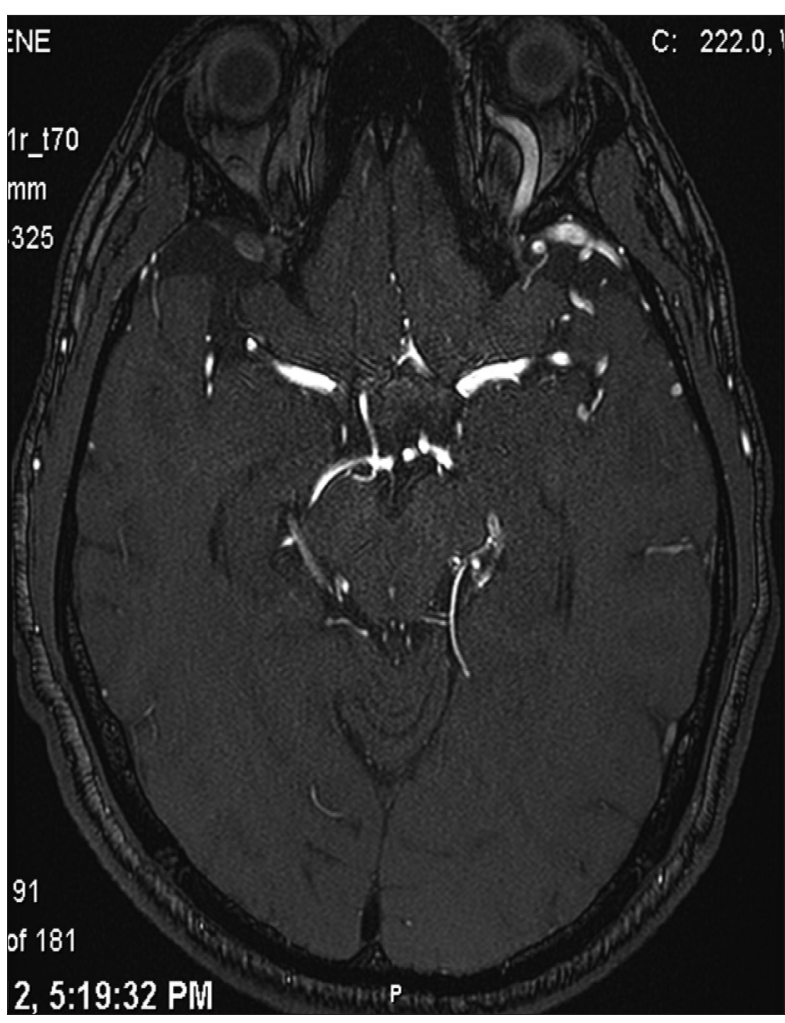

Figure 1. T2 weighted MRI Brain - Left carotid cavernous fistula, most likely direct (type A) with enlarged drainage vessels which included the left superior ophthalmic, left sphenoparietal and inferior petrosal sinuses.

\section{Case Report}

Thirty-two year old Hispanic male who was in a rear ended motor vehicle collision a day prior was admitted with complains of a severe throbbing headache in the left parietal region with no loss of consciousness but had nausea, dizziness, left eye redness but no retro-orbital pain and ringing sensation in the left ear. Patient had previous history of motor vehicle with transient loss of consciousness six years ago. Pertinent physical findings included mild left eye proptosis and chemosis with no neurological deficits. His pupils were $3 \mathrm{~mm}$ bilaterally, equal round and reactive to light with intact extraocular movements and no nystagmus. Tympanic membrane examination was normal. All cranial nerve were assessed and normal on examination. Rinne test revealed air conduction that is better than bone conduction and the weber test showed equal lateralization to both ears. No motor or sensory deficits to light touch, pain, position sense or vibration noted. All reflexes such as biceps, knee tendon and babinski sign were normal. No deficits in muscle strength ( $5 / 5$ all four extremities) or tremors. All laboratory work up on admission was within normal limits. 

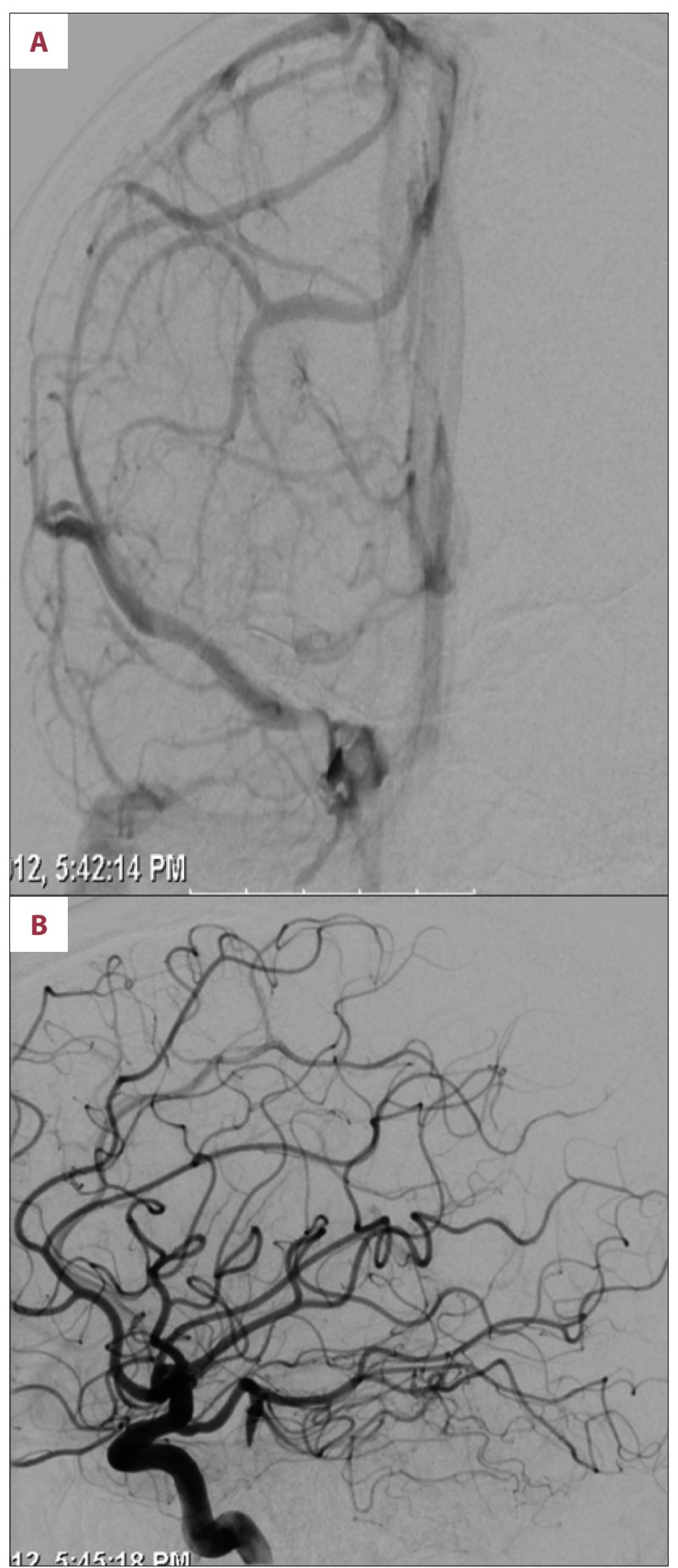

Computed Tomography of the brain showed an asymmetric bulging density of the left cavernous sinus with a dilated ipsilateral superior ophthalmic vein with increased density of the major intracranial vessels and dural venous sinuses. Magnetic Resonance Imaging (Figure 1) of brain revealed a left carotid cavernous fistula, most likely direct (type A) with enlarged drainage vessels which included the left superior ophthalmic, left sphenoparietal and inferior petrosal sinuses.

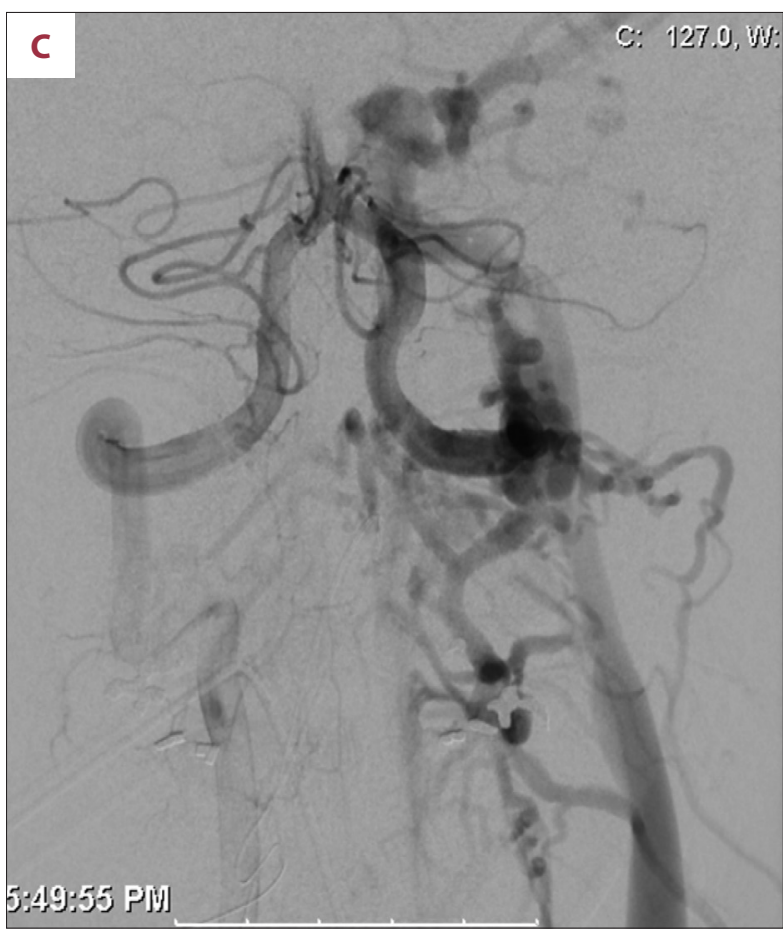

Figure 2A-C. Cerebral Angiogram - Basal dural arteriovenous fistula of the posterior fossa. The feeder vessels were mainly from the distal V3-V4 segment of the left vertebral artery and a lesser degree from a contribution from the left occipital artery.

Conservative treatment was recommended for fracture of the left lamina papyracea (medial orbital wall blowout fracture) that was present.

A cerebral angiogram (Figure $2 \mathrm{~A}-\mathrm{C}$ ) was performed before an attempted embolization of the arteriovenous fistula, but it was discovered that there was high flow skull basal dural arteriovenous fistula of the posterior fossa. The feeder vessels were mainly from the distal V3-V4 segment of the left vertebral artery and a lesser degree from a contribution from the left occipital artery. The left vertebral artery was noted to be significantly hypertrophic due to stealing blood flow from the contralateral right vertebral artery. There was insufficient cerebral blood flow into the distal basilar tip and its terminal branches. The basilar tip and its terminal branches were perfused through prominent bilateral posterior communicating arteries. Drainage was mainly into the suboccipital plexus and left jugular bulb. Retrograde drainage from the left jugular bulb into the inferior petrosal sinus, cavernous sinus and retrograde drainage into a hypertrophic left superficial middle cerebral vein that subsequently drain cortical veins of the dorsolateral aspect of the left cerebral hemisphere were noted. There was also retrograde cortical venous drainage from the cavernous sinus into the superior ophthalmic vein and angular vein down to the facial vein and drainage into the deep 
venous system through the left lateral vein of Rosenthal into the vein of Gallen and straight sinus.

The patient had a successful embolization of the high flow dural arteriovenous fistula of the posterior fossa mainly fed by the V3-V4 segment of the left vertebral artery and a less degree by the left occipital artery. A total of seven coils were used for transvenous and transarterial embolization using the assistance of a $7 \times 10 \mathrm{~mm}$ Scepter $\mathrm{C}$ balloon. At the end of the procedure there was very minimal and very late residual filling of the cavernous sinus. There was excellent blood flow throughout the vertebra-basilar system, and the patient was later discharged home one day postoperative in stable condition.

Unfortunately, the patient failed to follow up in clinic for scheduling of a repeat cerebral angiography due to lack of financial support. Patient eventually presented to the neurology clinic eight months after his endovascular embolization procedure. He complained of relapsing tinnitus and a sensation of fullness in the left mastoid area for the past week. Patient was admitted and subsequently brought to the neuroangiography suite for a diagnostic cerebral angiogram with the intention to treat the residual arteriovenous fistula. A successful embolization was achieved of the extracranial arteriovenous fistula arising from the V3 segment of the left vertebral artery using a total of three 18 Microvention coils. The next day, the patient was not complaining of any tinnitus in left ear or any pain at the mastoid region. He was therefore discharged home with follow in neurology clinic in two weeks.

\section{Discussion}

Patients with DAVFs may be completely asymptomatic. The symptoms experienced by the patient mainly depend on the location of the fistula. A cranial DAVF may present with pulsatile tinnitus that is described as a whooshing sound heard by the patient. Patients with drainage via the ophthalmic vein often display ocular symptoms, including conjunctival congestion, exophthalmos, intracranial murmur, visual disturbance, and oculomotor paralysis [8]. It may also present with an isolated but persistent or progressive headache, or symptoms and signs of a brain hemorrhage including sudden severe headache. In this case, the branches that supplied the meningeal artery of the DAVF were from the posterior cranial fossa dural branches from the vertebral and occipital arteries.

Imaging by $\mathrm{CT}, \mathrm{CT}$ angiography (CTA), MRI, and cerebral angiography play an important role in the investigation of patients with DAVFs. For a cranial DAVF, MRI is very helpful in defining the DAVF as CT may show only hemorrhage and not the fistula. The gold-standard for detection and characterization of a DAVF is a cerebral angiography on which a DAVF often appears as a complex vascular abnormality made up of abnormally large and tortuous dural arteries, and a large serpentine draining vein. The common angiographic findings seen are feeders from pial and dural branches, AV shunting, venous ectasia, stenosis, calcifications, impaired drainage of cerebral parenchyma with delayed venous drainage, and cortical venous collaterals [9].

DAVF is a treatable disease, but is undetectable in some cases and this eventually leads to irreversible disease because of the diagnostic delay [10]. The reported annual morbidity and mortality rates of aggressively presenting DAVFs may vary widely, ranging from $1.8-20 \%$ per year. Many DAVFs remain stable and do not change on follow-up and some may involute spontaneously. The need for treatment becomes urgent if the cortical venous drainage is seen on the cerebral angiogram or if a patient has experienced a rupture or other significant neurological effects of the DAVF.

At present, the two main ways of treating a DAVF are through open surgery or endovascular surgery. With the advent of more advanced interventional devices and techniques during the past decade, increasing reliance is being placed on endovascular therapy as a first-line treatment. Endovascular surgery involves a catheter-based technique for embolization of the lumen of arteries feeding the DAVF, or directly into the vein draining the DAVF. The goal of treatment for intercavernous and carotid-cavernous sinus fistulas is to block the fistulous communications and thus decrease the hemodynamic burden in the cavernous sinus [11]. This can be accomplished either by occlusion of arterial feeders and proximal venous drainage through a transarterial approach or by retrograde transvenous occlusion of the sinus harboring the fistula. The transvenous approach is regarded to be the ideal treatment method for cavernous sinus DAVFs, with the ability to cure a fistula in a single session and with a high success rate [12]. Healthcare providers should be aware of the typical symptoms and radiographic findings of a DAVF. A carotid cavernous fistula (CCF) could easily be mistaken for a DAVF on radiographic imaging. However, a cerebral angiography will be instrumental in distinguishing a DAVF from a CCF.

\section{Conclusions}

Performing a cerebral angiogram is pertinent in any patient that on initial imaging, such as on CT or MRI brain demonstrates the possibility of an arterio-venous fistula. We initially suspected that our patient to have a carotid-cavernous fistula but upon cerebral angiogram was actually diagnosed with a dural arterio-venous fistula in the posterior fossa venous system. The clinical presentation of DAVF is highly variable and depends on the location of the supplying and draining vessels as well as the presence of complications [13]. The most 
feared complication of a cranial DAVF is brain hemorrhage. It is very important to recognize the typical findings of patients presenting with a DAVF then quickly proceeding with a cerebral angiogram to determine the exact location of the fistula and the appropriate treatment plan. By diagnosing and treating a DAVF as early as possible, the associated fatal complications can be averted. It is also important for patients to have routine follow up post endovascular embolization to detect any residual arterio-venous fistula as was the case in our patient.

\section{References:}

1. Haraguchi K, Izumi T, Matsubara N et al: Mechanism of the formation of dural arteriovenous fistula: the role of the emissary vein. Interv Neuroradiol, 2011; 17(2): 195-202

2. Baik SK, Han MH, Jung C et al: Intraosseous cranial dural arteriovenous fistula treated with transvenous embolization. Am J Neuroradiol, 2009; 30(6): 1173-77

3. Fiorella DJ, Gonugunta V, Kelly ME et al: Marginal sinus arteriovenous fistulas mimicking carotid cavernous fistulas: diagnostic and therapeutic considerations. Am J Neuroradiol, 2007; 28(10): 1915-18

4. Borden JA, Shucart, WA, Wu JK: A proposed classification for spinal and cranial dural arteriovenous fistulous malformations and implications for treatment. J Neurosurg, 1995; 82(2): 166-79

5. Kuker W, Padmanabhan R, Stacy R, Wimalaratna S: Dural arteriovenous fistula causing primary intraventricular haemorrhage. Br J Radiol, 2008, 81(962): e44-e47

6. Abud DG, Abud TG, Colli BO et al: Dural arteriovenous fistulas with direct cortical venous drainage treated with Onyx: a case series. Arquivos de Neuro-Psiquiatria, 2010; 68(4): 613-18

\section{Disclosures}

All participated authors in this study declare no financial, professional or personal conflicts.

\section{No grant support was received for this case report.}

All the above authors mention above were involved in the preparation of this manuscript.

7. Ahn HS, Choi SJ, Chung SJ et al: New cconcept in cavernous sinus dural arteriovenous fistula: correlation with presenting symptom and venous drainage patterns. Stroke, 2005; 36(6): 1134-39

8. Arai $\mathrm{H}$, Melake $\mathrm{M}$, Oishi $\mathrm{H}$ et al: Transvenous embolization of dural carotid cavernous fistulas: a series of 44 consecutive patients. Am J Neuroradiol, 2010; 31(4): 651-55

9. Gupta AK, Periakaruppan AL: Intracranial dural arteriovenous fistulas: a review. Indian J Radiol Imaging, 2009; 19(1): 43-48

10. Chang $\mathrm{KH}, \mathrm{Han} \mathrm{MH}$, Kang HS, Kwon BJ: MR imaging findings of intracranial dural arteriovenous fistulas: relations with venous drainage patterns. Am J Neuroradiol, 2005; 26(10): 2500-7

11. Nishizawa S, Sugiura. Arteriovenous fistula involving the inferior petroclival vein - case report. Neurologia Medico-Chirurgica, 2011; 51(1): 45-47

12. Cloft HJ, Lanzino G, Loumiotis I: Intercavernous sinus dural arteriovenous fistula successfully treated with transvenous embolization. a case report. Interv Neuroradiol, 2011; 17(2): 208-11

13. Bulters DO, Culliford D, Mathad $\mathrm{N}$ et al: The natural history of cranial dural arteriovenous fistulae with cortical venous reflux - the significance of venous ectasia. Neurosurgery, 2012: 70(2): 312-18 